\title{
Local recurrence after conserving breast therapy: A retrospective study
}

\author{
AM El-Anwar, ${ }^{a} M D ;$ M Shinawi, ${ }^{a} M D ;$ A Nafae, ${ }^{a} M D ;$ M Alaa Osman, ${ }^{a} M D$; \\ Hany Abd El-Aziz, ${ }^{b}$ MD; Hisham El-Ghazaly, ${ }^{b} M D$
}

a) Department of General Surgery, Ain Shams University, Cairo, Egypt.

b) Department of Radiotherapy, Ain Shams University, Cairo, Egypt.

\begin{abstract}
Breast conservative therapy $(B C T)$ is the preferred treatment for early breast cancer and carries low local recurrence (LR) rates. Proper follow up is mandatory and includes periodic clinical examination, mammography and ultrasound to detect early recurrence. This study aims at evaluating the results of breast conservative therapy especially as regard local recurrence.

Methods: Between January 2000 and July 2008, 241 consecutive female patients with early breast cancer were surgically treated by conservation surgery plus radiotherapy. Two patients had bilateral breast cancer and were subjected to preservation surgery on both sides in the same setting. The median age of patients was 48 years (range 23-76). Two hundred twenty four cases were treated by wide local excision and axillary clearance (level I\&II) and 19 cases utilized the down staging policy which entails preoperative chemotherapy followed by preservation procedure. Patients were followed after surgery from one to 10 years (average 5.5yrs).

Those who developed suspicious lesions on mammography or ultrasound were subjected to magnetic resonance imaging (MRI) and then all suspicious lesions were subjected to biopsy and histopathologic examination.

Results: During follow-up, 62 out of 241 cases showed abnormal mammographic findings and were all subjected to magnetic resonance imaging (MRI) and then biopsy of all suspicious lesions.

Local recurrence was detected in 12 of them (4.9\%). For 11 cases, LR was the only event and one case showed coincident LR and distant metastasis. The recurrences detected were true $(n=7)$ or marginal $(n=3)$, which arise within or adjacent to the excision area, respectively. Only 2 recurrences were located elsewhere in the breast $(n=2)$.

In the present study, patients with large breasts were found to have a lower risk of local recurrence compared to patients with small breasts; highly statistically significant relation $(p<0.01)$. Statistically significant relation was also found between young age and the risk of $L R(p<0.05)$.

In the present study, patients with tumor size $<2 \mathrm{~cm}$ were found to have a lower risk of local recurrence compared to patients with tumor size $>2 \mathrm{~cm}$, statistically significant relation ( $p=0.017)$. Statistically significant relation was also found between positive lymph nodes (+ve LNs) and the risk of $L R(p<0.01)$.

Four patients with LRs underwent salvage mastectomy, and the other 8 patients received wider local excision and adjuvant therapy. No mortalities were detected in patients with LR.

In conclusion, breast conserving therapy is an appropriate primary therapy for early breast cancer, surgeons should be aware of the mammographic findings following such a surgery. Young age, breast size, tumor size and lymph status should be considered in planning optimal treatment for breast cancer. Detection of biological risk factors for types of $L R$ would be fruitful to distinguish unfavorable LRs that need systemic therapy from favorable LRs which could be treated only locally.
\end{abstract}




\section{Introduction:}

The long term survival rate among women who undergo breast conserving surgery is the same as that among women who undergo radical mastectomy, therefore breast conserving surgery is currently considered the treatment of choice for women with relatively small breast cancers, ${ }^{1}$ and the recurrence rate reported in these patients is about $1-2 \%$ per year. ${ }^{2,3}$ Long term survival of patients with new malignancy after conservative treatment improves with early detection. ${ }^{4}$

However, a number of risk factors are associated with an increased likelihood of local recurrence after breast conserving therapy (BCT). The most established risk factors are young age, ${ }^{5}$ the presence of disease at resection margin, ${ }^{6}$ multicentric disease ${ }^{7}$ and vascular invasion. ${ }^{8}$ Extensive intraductal component (EIC) has been much discussed as a risk factor, but seems to have little prognostic value if negative margins can be obtained. ${ }^{9}$

The diagnostic evaluation of the treated breast is unfortunately still a challenge because post-treatment changes of breast tissue can show great variability, hiding or mimicking recurrent lesions. Changes following breast conserving surgery can include hematoma, seroma, fat tissue necrosis, scar tissue development and dystrophic calcifications. Changes after radiotherapy can include vascular dilatation, capillary damage, microcirculatory changes and edema. The association of these changes after breast conserving surgery and irradiation make the interpretation of clinical examination and mammography very difficult because of focal thickening, decreased compressibility and increased density at the surgical site. ${ }^{10}$

Clinical examination, mammography or ultrasonography can raise a suspicion but an additional evaluation is frequently mandatory to avoid unnecessary biopsy or surgery. Several recent studies have shown the important role of breast magnetic resonance imaging (MRI) imaging for detection of recurrent lesions in patients treated with conservative breast surgery and radiotherapy. ${ }^{10}$

Despite the importance of achieving a negative margin in the breast conserving procedure, a negative margin may under predict residual disease, as demonstrated by the reanalysis of the National Surgical Adjuvant Breast and Bowel Project. ${ }^{11}$

Consequently, many techniques have been evolved in an attempt to increase the detection of residual disease after breast conserving surgery such as, re-excision of positive margin, examination of bed biopsies of the cavity and cavity shaving.

\section{Aim of the work:}

This study was intended to evaluate the results of breast conserving therapy between January 2000 and July 2008, especially with respect to the local recurrence (LR) rate.

\section{Patients and methods:}

Between January 2000 and July 2008, 241 female patients presented with stage I or II breast cancer were treated by BCT in Ain Shams University Hospitals. The median age of patients was 48 years (range 23-76). Two patients had bilateral breast cancer and were subjected to preservation surgery on both sides in the same setting. Diagnosis of breast cancer was by mammography and fine needle aspiration cytology, Tru-cut biopsy or excision biopsy.

All patients were subjected to frozen section during surgery primarily for diagnosis of malignancy if not already diagnosed and to document free margins around the excised mass Figure(1).

Two hundred twenty four cases were treated by wide local excision and axillary clearance (level I\&II), 19 cases utilized the down staging policy which entails preoperative chemotherapy followed by preservation procedure Table(1).

\section{Surgery:}

Breast conserving surgery is performed as a wide local excision and axillary clearance (level I\&II). Wide local excision entails excision of tumor with at least $2 \mathrm{~cm}$ of the surrounding macroscopic normal breast tissue and excision of axillary nodes level I\&II Figure(2). Following excision of the primary tumor the entire cavity wall surrounding the lump was excised completely producing a specimen shaped like a ring termed "cavity shaving". The thickness of the cavity shaving 
was approximately $1 \mathrm{~cm}$. The wide local excision specimen and the cavity shaving

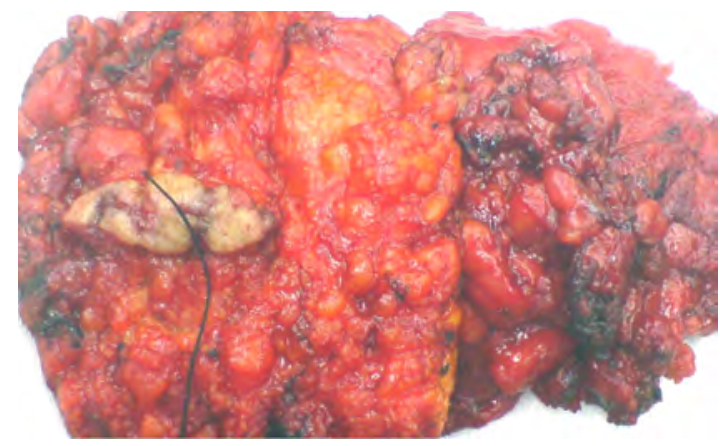

Figure (1): Excised mass with safety margin labeled for frozen section.

\section{Radiotherapy:}

Radiotherapy was administered in a standard way, using two tangential fields directed at the whole breast and chest wall. For most, a boost dose was given to the tumor bed using a 12$17-\mathrm{MeV}$ electron beam directed at a $90^{\circ}$ angulation's at the skin. If electron was not available the boost was given using a photon beams $\left(\mathrm{Co}^{60}\right)$ 10-16 Gy. The radiation doses given were 50 Gy in 5 weeks (fraction dose 2 Gy, 5 days a week) for the whole breast and 14-16 Gy for the boost.

\section{Quality control study:}

The radiotherapy treatment was revised in case of local relapse to exclude the possibility of an inadequate boost. The adequacy of the boost, considering radiation energy, margins of the radiation field, and location of the boost, was checked by reviewing the preoperative mammogram and the simulator films. Also, the postoperative mammogram was collected and reviewed. The pathology report and slides of the tumor excision was taken into account, as was the operation report.

\section{Follow-up:}

\section{Figure(3-5)}

Patients were followed after surgery from one to 10 years (average 5.5yrs). Follow-up was performed every six months for two years then every year later by mammography, breast ultrasound, abdominal ultrasound, chest $\mathrm{x}$-ray specimen were submitted for histopathological analysis Figure(1).

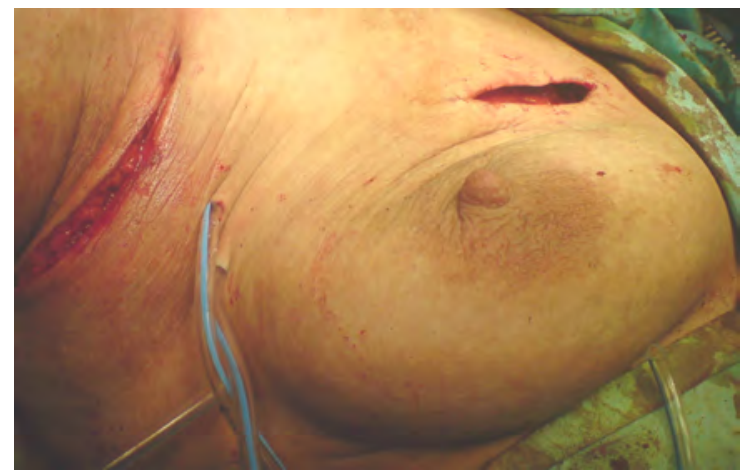

Figure (2): Patient with BCT and axillary clearance.

and serum tumor markers. Bone scan was performed every two years. Local recurrence was defined as the appearance of any morphologically verified ipsilateral new tumor, invasive or in situ, in the operated breast parenchyma or in the overlying skin, before or at the same time of distant metastasis. Patients were considered to have a LR after histological or cytological confirmation.

\section{Results:}

In total, 241 patients were studied with 243 tumors, with the histopathological characteristics of tumor specimens listed in Table(2). Relatively many young women (<50 years old: $56 \%$ ) and small tumors $(51 \% \mathrm{pTl})$ with mostly negative lymph nodes (64\% pNO) were included in our study. The median age of patients was 48 years (range 23-76). The median follow up was 5.5 years.

During follow-up, 62 cases showed abnormal mammographic findings and were all subjected to magnetic resonant imaging and then all suspicious lesions were subjected to biopsy and histopathologic examination Table $(3,4)$. Local recurrence was detected in 12 out of 241 cases Figure(6,7). For 11 cases LR was the only event, whereas the last case showed coincident LR and distant metastasis. Eighteen out of 241 patients had developed distant metastasis with mortality involving 17 of them Table(7). 
Local recurrence risk and detailed analysis of patients with a local recurrence:

The median time of development of LR after surgery was 42 months. Most of the LRs were located within the excised area, described as true recurrences $(n=7)$, or adjacent to the excision area, described as marginal recurrences $(n=3)$. Recurrences located elsewhere in the breast $(\mathrm{n}=2)$.

Younger age was a risk factor for developing a LR Table(5). Patients between the ages of 28 and 42 years old had a 2 times higher risk of developing local relapse compared to patients between the ages of 48 and 54 years old. In patients more than 54 years old, no local relapses were detected. Statistically significant relation was detected between the age distribution and local recurrence by Fisher exact test $(\mathrm{p}<0.05)$.

LR risk of patients with small breast was 5 times higher compared to those with large breast with highly statistically significant difference in between by Fisher exact test $(\mathrm{p}<0.01)$ Table $(6)$.

Patients with tumor size $<2 \mathrm{~cm}$ were found to have a lower risk of local recurrence compared to patients with tumor size $>2 \mathrm{~cm}$ with local recurrence, statistically significant relation by Fisher exact test $(\mathrm{p}=0.017)$ Table $(\mathbf{8})$. Statistically significant relation was also found between positive lymph nodes (+ve LNs) and the risk of LR by Fisher exact test $(\mathrm{p}<0.01)$ Table(9).
Histopathological reports for patients with LR were revised for ER and PR receptors where 7 patients $(58 \%)$ showed +ve receptors and 5 patients $(41 \%)$ showed -ve receptors.

\section{Radiotherapy revision: Quality control study:}

A possible cause of $\mathrm{LR}$ is inadequate radiotherapy planning. Therefore, radiotherapy planning was revised in all patients with LR. Of these, 10 patients were considered to be adequately treated with radiation therapy. In two patients, the postoperative mammograms showed a field of malignant micro calcifications, still present in the direct neighborhood of the primary tumor bed. The boost radiation field did not fully encompass these micro calcifications.

\section{Surgical margin revision: Quality control study:}

We examined the relationship between the margin status and the LR retrospectively. Only one patient with LR was found to have a positive margin. The pathological slides were not available for revision in 3 patients, in such patients we rely on the pathological reports.

\section{Treatment of local recurrences and survival} after LR:

Four patients with LR underwent salvage mastectomy, and the other 8 patients received wider local excision and adjuvant therapy.

Table (1): Surgical procedure performed in 241 cases.

\begin{tabular}{|l|l|}
\hline No. of cases & Procedure \\
\hline 224 & Wide local excision + Level I, II nodes \\
\hline 19 & $\begin{array}{l}\text { Down staging, preoperative chemotherapy 3 months } \\
\text { (wide local excision + Level I, II nodes) }\end{array}$ \\
\hline
\end{tabular}

N.B. 2 cases were bilateral.

Table (2): Histopathological characteristics of tumor specimens (243 specimens).

\begin{tabular}{|l|c|c|c|c|c|}
\hline \multirow{2}{*}{ Tumor size } & \multirow{2}{*}{$\begin{array}{c}\text { No. of } \\
\text { specimens }\end{array}$} & \multicolumn{2}{|c|}{ LN status } & \multicolumn{2}{c|}{ Tumor grade } \\
\cline { 3 - 6 } & & -ve Node & +ve Node & Grade I & Grade II \\
\hline Less than $2 \mathrm{~cm}$ & 124 & 90 & 34 & 82 & 42 \\
\hline $2-3 \mathrm{~cm}$ & 77 & 51 & 26 & 47 & 30 \\
\hline $3-4 \mathrm{~cm}$ & 42 & 14 & 28 & 15 & 27 \\
\hline
\end{tabular}


Table (3): Abnormal mammographic findings among 241 patients.

\begin{tabular}{|c|c|c|}
\hline No. of patients & $\begin{array}{c}\text { Mammographic } \\
\text { Findings }\end{array}$ & Time of Detection (months) \\
\hline 20 & Scar retraction with specula & $6-12$ \\
\hline 14 & Well- circumscribed density & $4-12$ \\
\hline 6 & Local calcifications & $24-30$ \\
\hline 10 & Speculated masses & $12-18$ \\
\hline 12 & Discrete masses & $18-36$ \\
\hline
\end{tabular}

Table (4): Abnormal mammographic findings and their histopathological nature (241 PTS).

\begin{tabular}{|c|c|c|}
\hline No. of patients & $\begin{array}{c}\text { Mammographic } \\
\text { Findings }\end{array}$ & Biopsy Result \\
\hline 20 & Scar retraction with specula & Extensive fibrosis \\
\hline 14 & Well- circumscribed density & $\begin{array}{c}\text {-Seromas - One of them contained } \\
\text { pyogenic granuloma. Figure(8) }\end{array}$ \\
\hline 6 & Local calcifications & $\begin{array}{c}\text { 3 local recurrence, 3 Dystrophic } \\
\text { calcification in fat necrosis }\end{array}$ \\
\hline 10 & Speculated masses & 9 Fibrosis, 1 Local recurrence \\
\hline 12 & Discrete masses & 4 Fibrosis, 8 Local recurrence \\
\hline
\end{tabular}

Table (5): Statistically significant relation was detected between the age distribution and local recurrence by Fisher exact test $(p<0.05)$.

\begin{tabular}{|l|c|c|}
\hline Age group & Mean + SD & No. of patients \\
\hline $28-42$ years & $29.5 \pm 10.6$ & 8 patients \\
\hline $48-54$ years & $48.2 \pm 15.2$ & 4 patients \\
\hline$>54$ years & $52 \pm 12.3$ & - \\
\hline
\end{tabular}

Table (6): Shows that cases with small breast had a higher recurrence rate compared to those with large breast with highly statistically significant difference in between by Fisher exact test $(p<0.01)$.

\begin{tabular}{|l|c|c|}
\hline Breast size & No. of patients & Percentage \\
\hline Small breast & 10 patients & $(83.3 \%)$ \\
\hline Large & 2 patients & $(16.7 \%)$ \\
\hline
\end{tabular}


Table (7): Distant metastasis among 241 patients.

\begin{tabular}{|c|c|c|c|c|c|}
\hline $\begin{array}{l}\text { No. of } \\
\text { patients }\end{array}$ & Age & Site of metastasis & Free survival & Overall survival & Mortality \\
\hline 7 & $40 \pm 3 \mathrm{ys}$. & Liver & $24 \pm 4 \mathrm{~ms}$. & $36 \pm 3 \mathrm{~ms}$. & 7 \\
\hline 6 & $50 \pm 2 \mathrm{ys}$. & Bone(Spine) & $28 \pm 5 \mathrm{~ms}$. & $48 \pm 5 \mathrm{~ms}$. & 5 \\
\hline 3 & $35 \pm 2 \mathrm{ys}$. & Pleural effusion & $20 \pm 4 \mathrm{~ms}$. & $30 \pm 6 \mathrm{~ms}$. & 3 \\
\hline 2 & $35 \mathrm{ys}$. & Brain & $24 \pm 3 \mathrm{~ms}$. & $30 \pm 3 \mathrm{~ms}$. & 2 \\
\hline
\end{tabular}

Table (8): Shows that cases with tumor size between 2-4 cm had higher recurrence rate compared to those with tumor size less than $2 \mathrm{~cm}$ with highly statistically significant difference in between by Fisher exact test ( $p=0.017)$.

\begin{tabular}{|l|c|}
\hline Tumor size & Local recurrence \\
\hline$<2 \mathrm{~cm}$ & $2 / 12(16.6 \%)$ \\
\hline $2-4 \mathrm{~cm}$ & $10 / 12(83.3 \%)$ \\
\hline
\end{tabular}

Table (9): Shows that cases with positive lymph nodes had higher recurrence rate compared to those with negative lymph nodes with highly statistically significant difference in between by Fisher exact test $(p<0.01)$.

\begin{tabular}{|c|c|c|}
\hline LN status & $\begin{array}{c}\text { +ve } \\
\boldsymbol{n}=\mathbf{8 8}\end{array}$ & $\begin{array}{c}\text {-ve } \\
\boldsymbol{n}=\mathbf{1 5 5}\end{array}$ \\
\hline LR & $9 / 12(75 \%)$ & $3 / 12(25 \%)$ \\
\hline
\end{tabular}



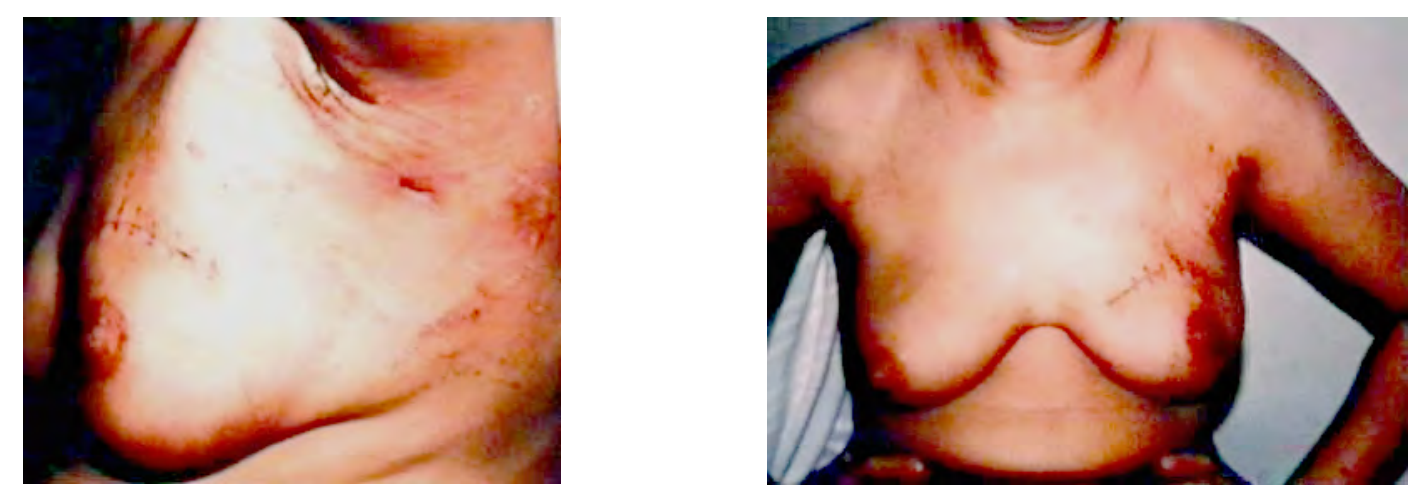

Figure (3): Follow up after BCT.
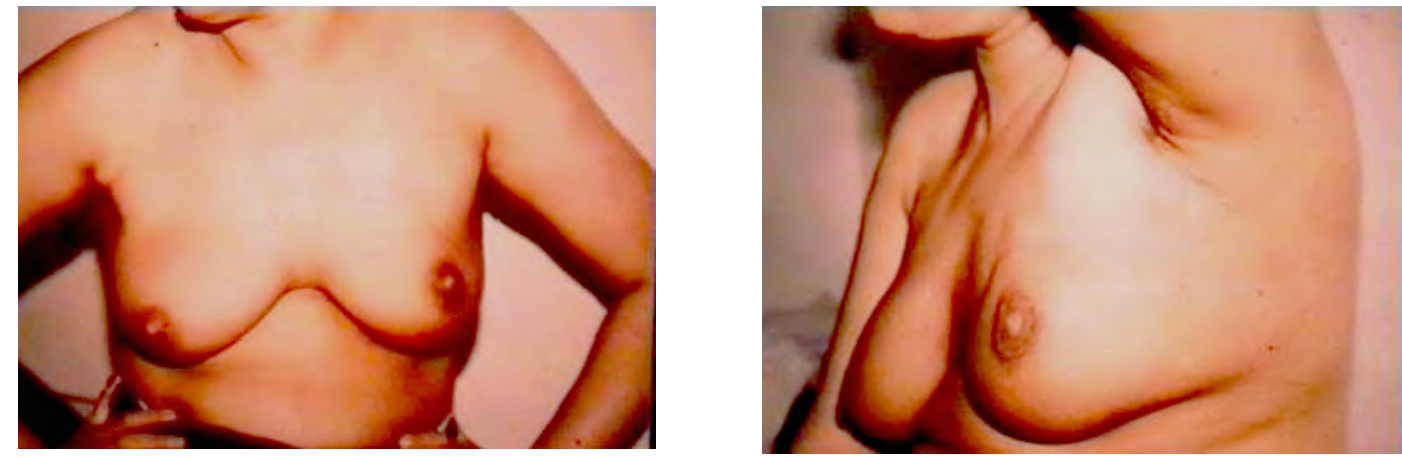

Figure (4): Follow up after BCT.
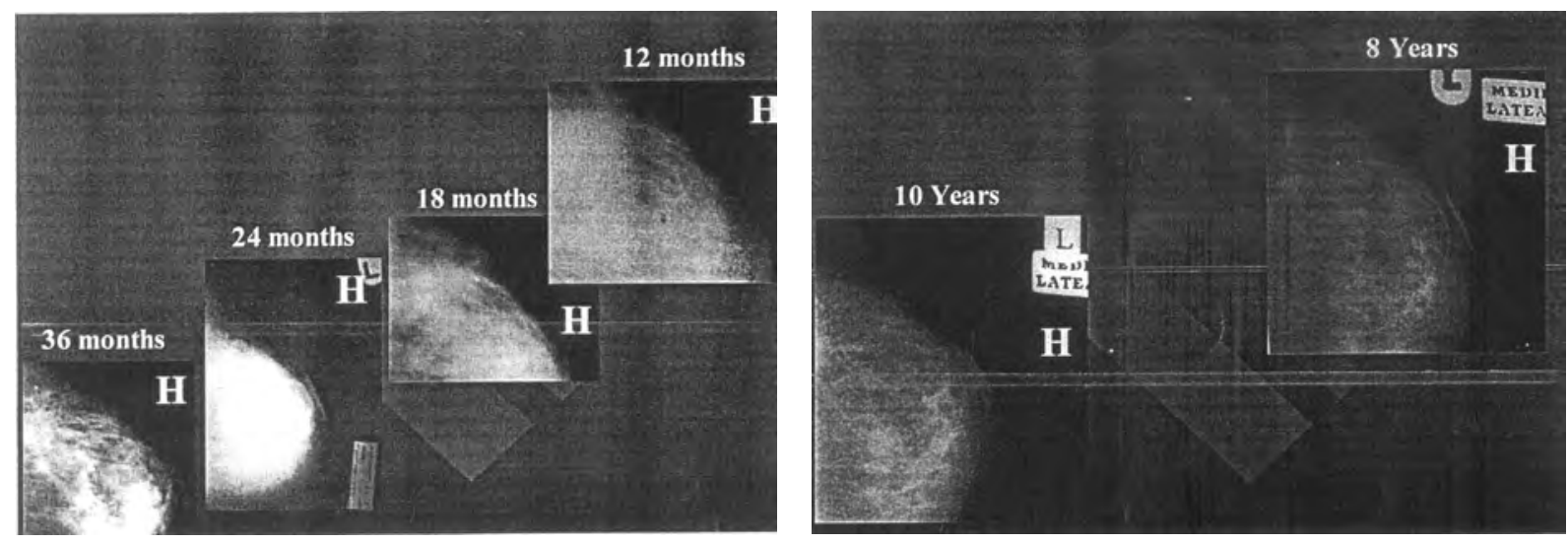

Figure (5): Serial mammography showing 10 years follow up with no local recurrence. 

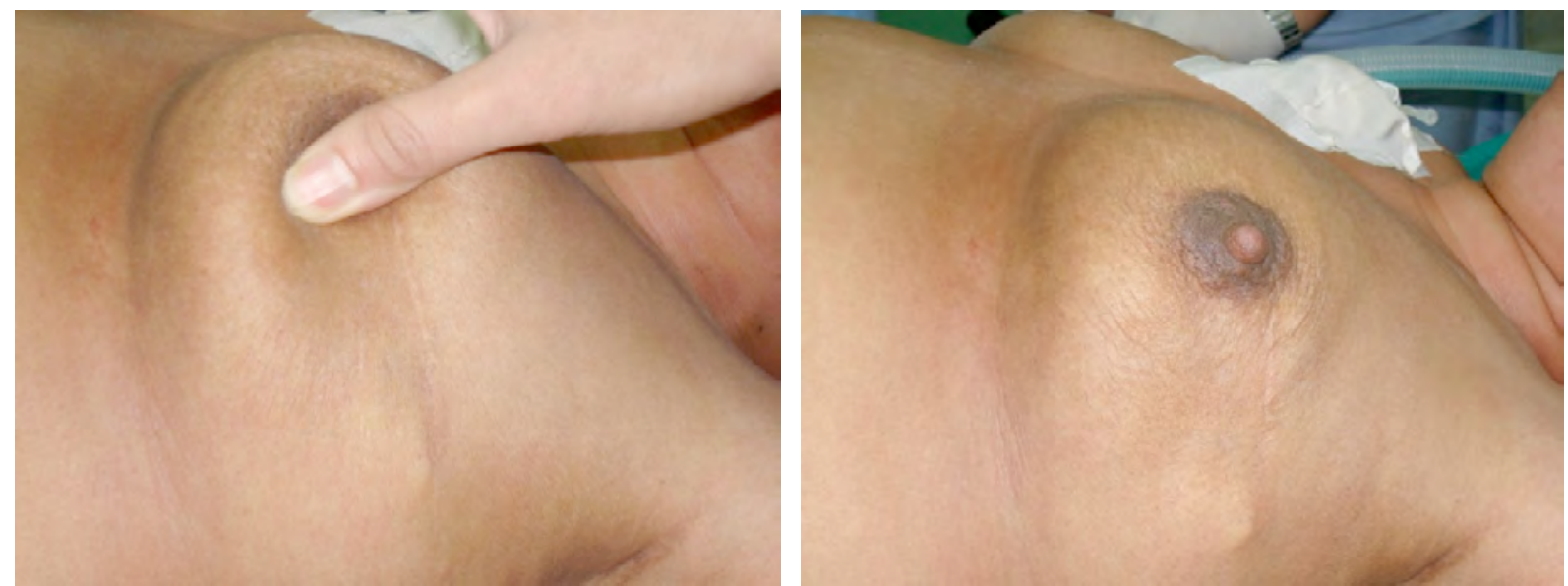

Fig. (6): A case of local recurrence after BCT.
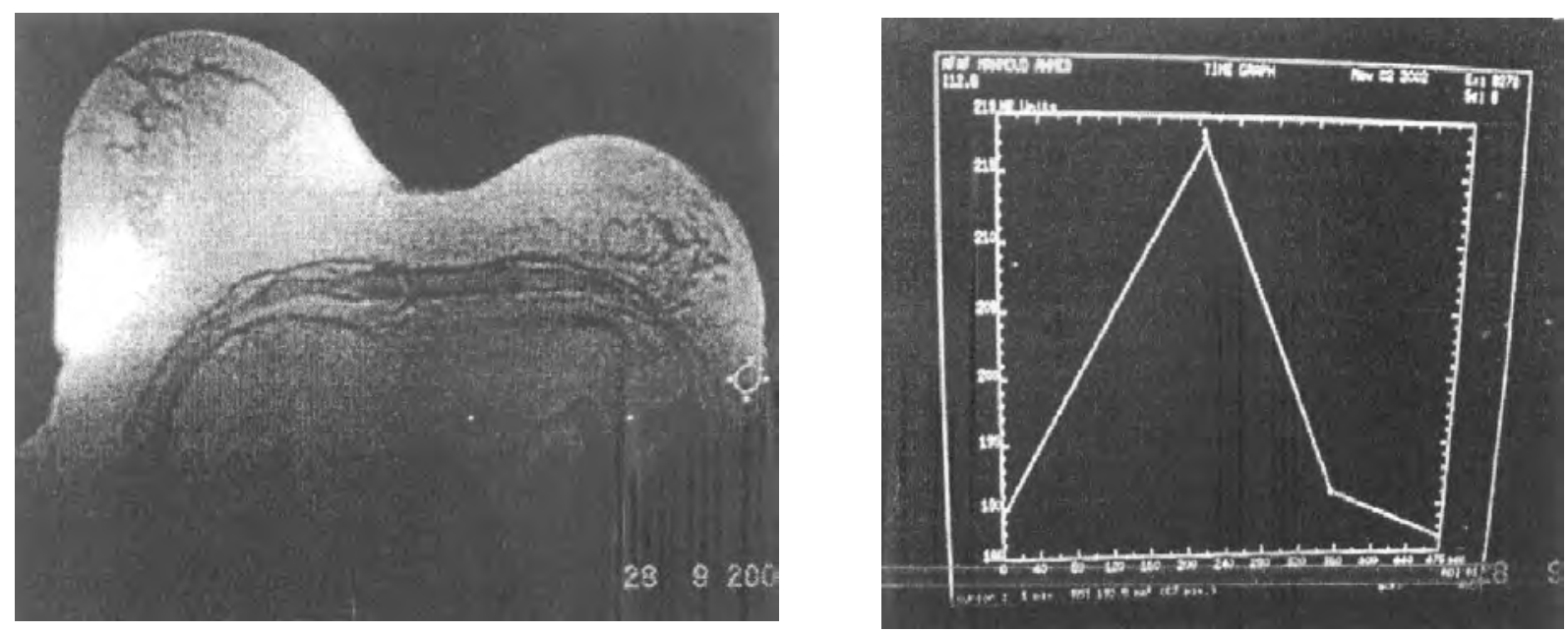

a) MRI mammography for patient with local recurrence.

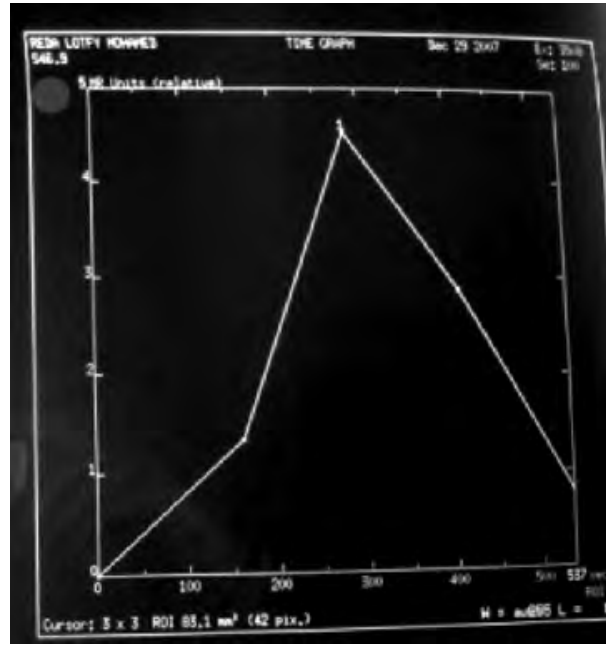

b) Post gadolinium curve for the same patient with local recurrence.

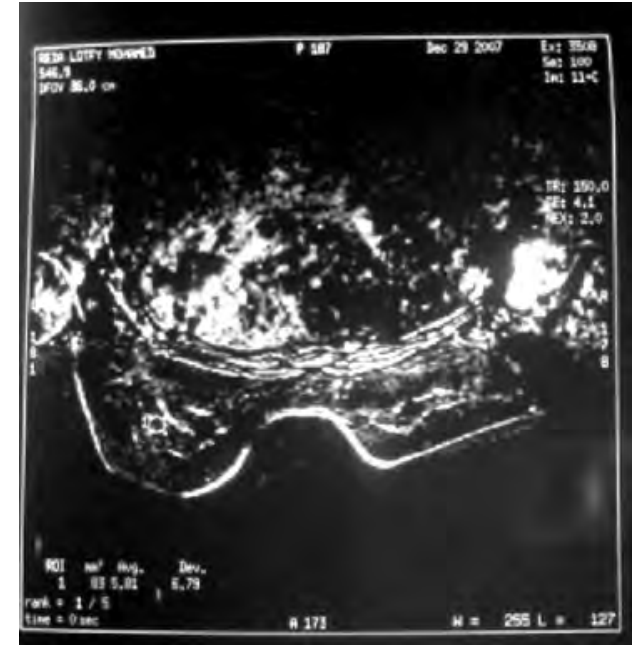

c) MRI mammography for patient with local recurrence.

d) Post gadolinium curve for the same patient with local recurrence.

Figure (7): MRI mammography showing local recurrence. 


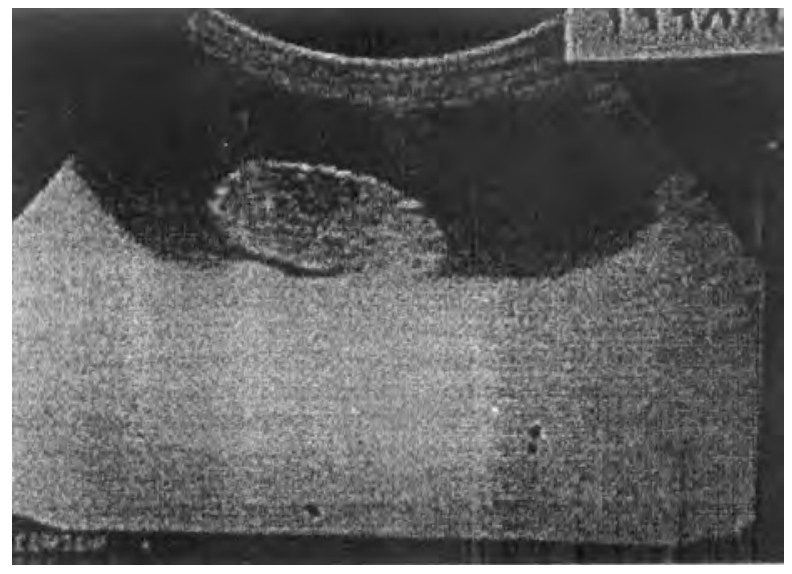

a) Ultrasonography.

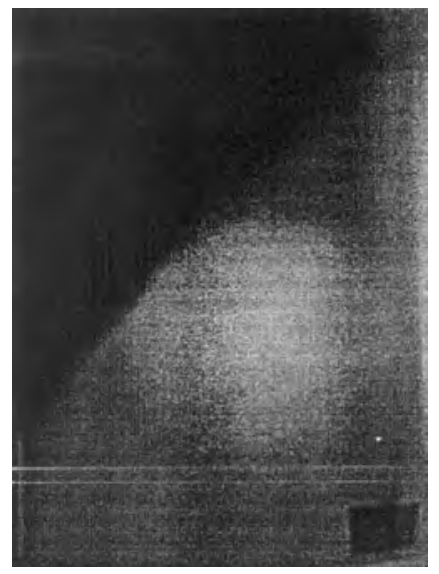

b) Mammography.

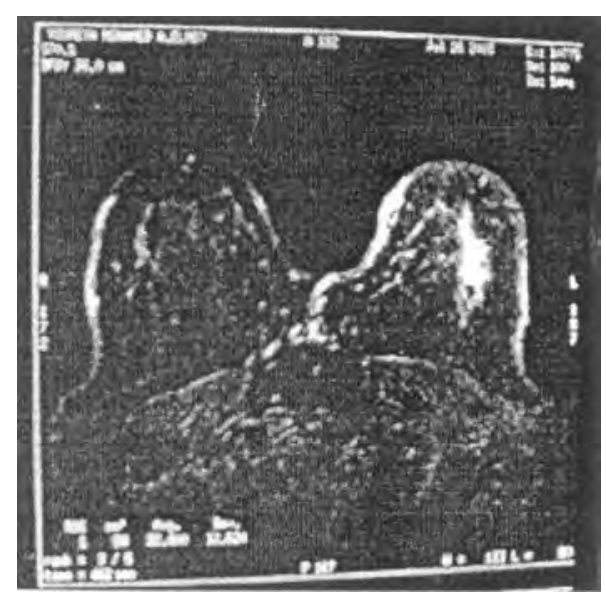

c) MRI mammography.

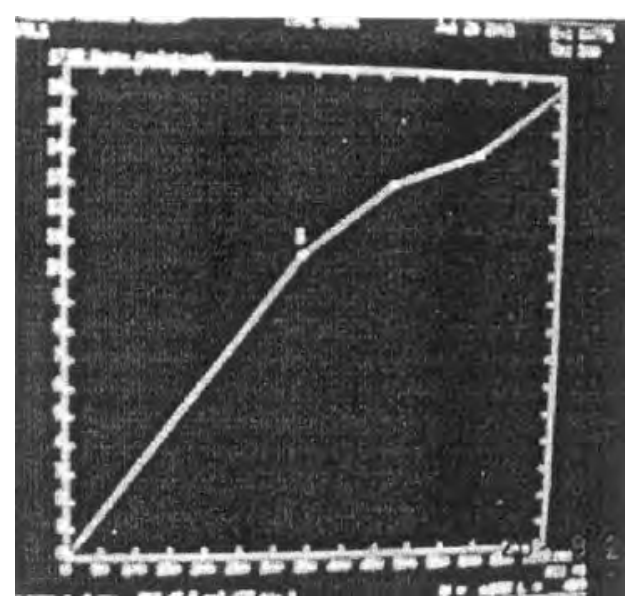

d) Post gadolinium curve.

MRI mammography with no local recurrence.

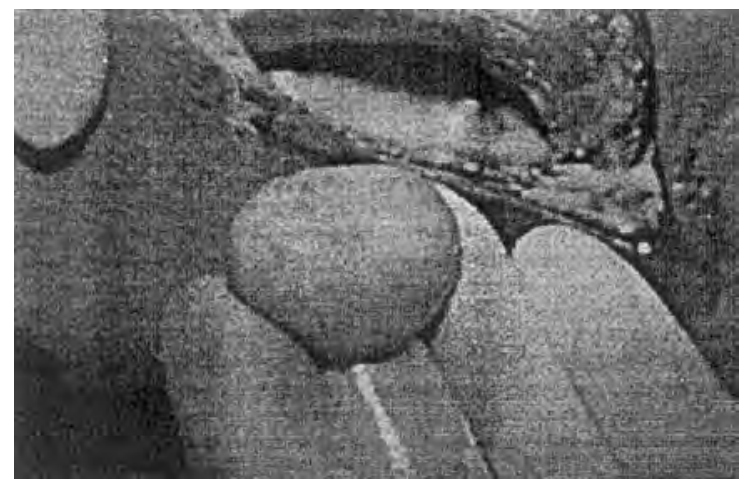

e) Excision of granuloma.

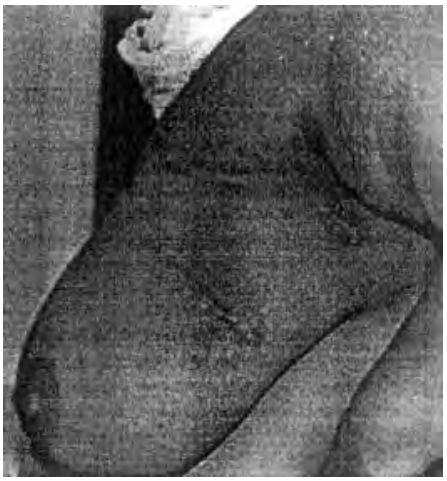

f) After removal.

Figure (8): Seroma containing biogenic granuloma. 


\section{Discussion:}

Despite the general acceptance of BCT for early stage breast cancer, a number of questions regarding this treatment have remained. We studied risk factors in BCT in 241 female patients with early breast cancer.

The incidence of loco regional breast cancer recurrences after breast conserving therapy in patients with operable breast cancer have been reported to occur in at least $1-2 \%$ of cases per year, 2,3 some authors ${ }^{12}$ found that LR rate was $6.3 \%$ at 5 year follow up. Touboul et al. ${ }^{13}$ observed 528 patients between 1976 and 1993 and noticed five- and ten- year recurrence rates of $6.8 \%$ and $14 \%$ respectively. In our study, 12 local recurrences appeared in 243 breast carcinomas $(4.9 \%)$, with a median interval between surgery and LR detection of 42 months. Comparable results were reported by Fredriksson et al. (2003). ${ }^{14}$ Slightly longer intervals have been reported by Elkhuizen et al. (1998). ${ }^{15}$

Variable LR rates could be explained by the different methods used, not only follow up is different, but also tumor therapy and namely surgical techniques used for excision of the primary tumor could be different. It is clear that variations in $L R$ rates after breast conserving surgery are positively affected by radiotherapy.

In most cases, LR was detected either by physical examination or by annual mammography. ${ }^{16}$ Several recent studies have shown the importance of breast magnetic resonance imaging for detection of recurrent lesions in patients treated with conservative surgery. 10,17

Magnetic resonance mammography (MRM) has high sensitivity, high specificity and high accuracy in differentiating physiologic changes of the scar from tumoral tissue. MRM multifactorial evaluation, based on both morphological features and time signal intensity curves of enhancing lesions, is related to significantly higher sensitivity and higher specificity than evaluation protocols based only on one morphological or enhancing feature. ${ }^{10,18}$

Preda et al. (2006) $)^{10}$ demonstrated the high sensitivity and high specificity of MRM in confirming or excluding recurrence at the prior lumpectomy site, after conservative surgery and radiation therapy, when recurrence was already suspected either by mammography (including the associated clinical examination) or by ultrasonography. MRM has shown an overall high negative predictive value $(98.8 \%)$ in the detection of breast cancer, including lesions not related to the surgical scar. This coincides with the results of the current study where the results of histopathology matched with the results of MRI.

Despite the high accuracy of MRM in detecting recurrence on the site of lumpectomy, its cost and low availability limit its use for the routine follow up of treated patients. ${ }^{19} \mathrm{In}$ some conditions, however, as in the presence of radio dense breasts and/or structural posttreatment changes, MRM represents an important diagnostic modality in support of the other traditional imaging modalities, and it can be considered conclusive when showing negative findings. ${ }^{10}$

The efficacy of MRI in detecting or excluding recurrence appear 18 months after radiation therapy because, after that time, enhancement of areas of radiation fibrosis is rare. $^{20}$

Of the LRs found in our study, 7 cases were true recurrences $(59 \%)$ and 3 cases were marginal recurrences $(25 \%)$, described as tumor relapse in or close to the excision area, respectively and 2 recurrences located elsewhere in the breast (16\%). Such results are in agreement with those recorded by Osborne et al. (1992). ${ }^{21}$ The interval to recurrence is supposed to be related to the location of the recurrences. True or marginal recurrences tend to occur earlier than recurrences located elsewhere in the breast. ${ }^{22}$ In our study, this was not observed. It seems that the routine follow-up as performed in our study is very useful to detect LR.

Our study confirms the importance of age at diagnosis as the most important risk factor for LR. ${ }^{23}$ We noticed that LR risk was not only higher in the young patients (28-42 years old), but also in the intermediate group (48-54 years old), compared to older patients ( $>54$ years old). It might be argued that, since younger patients are more likely to have an EIC than older patients, and in view of the fact that 
patients with an EIC are more likely than others to have considerable residual carcinoma, younger patients would appear to be at much higher risk of having a large residual tumor burden after a simple gross excision than older patients. ${ }^{5}$

A highly statistically significant relation was found between small breasts and high incidence of LR Table(6). Such findings could be attributed to a greater residual tumor burden in these patients because the volume of excised parenchyma related directly to the amount of residual carcinoma in the adjacent breast parenchyma. $^{24}$

A highly statistically significant relation was found between tumor size and high incidence of LR Table(8) and this matches with the study done by Mirza et al. ${ }^{25}$ They observed that patients with larger tumor diameter had higher risk for LR and locoregional recurrence (LRR) and metastases than patients with a smaller tumor size.

Another highly statistically significant relation was found between lymph nodes status +ve LN and LR which agrees with studies which confirmed that positive nodes increased either LR or LRR rate. ${ }^{26}$ In other studies ${ }^{13}$ axillary node status did not influence the rate of LR.

Receptor status (ER and BR) has also been reported as a risk factor increasing local recurrence. Bartelink et al. ${ }^{27}$ showed that the absence of progesterone receptor was a predictable factor for local recurrence. In our study we observed that local recurrence occurred more in women with negative as compared to positive estrogen and progesterone receptors.

We attempted to study the adequacy of the radiation therapy for patients with LRs. We are of the opinion that adequate radiation boosts were given in the majority of our patients. We can state that inadequate radiotherapy was not a factor resulting in LR in our patients.

Because we under no circumstances accept to conserve a breast with positive histological resection margin, the intimate relation between the positive resection margin and high incidence of LRR was not recorded in our patients. Such relation was highly significant in studies that offered little consideration for the negative resection margin as a prerequisite to perform breast conservation. 6,28

We treated all LRs surgically. Four patients underwent salvage mastectomy, and the other 8 patients received wider local excision and adjuvant therapy.

A very important issue in $\mathrm{BCT}$ is the possible role of LR on the course of the disease. Randomized trials have demonstrated that there were no difference in overall survival between patients with LR and patients without LR after $\mathrm{BCT}$, leading to the conclusion that LR does not result in poor prognosis. ${ }^{29}$ Other studies, however, described LR after BCT to be associated with poor prognosis. ${ }^{30}$ The proposed explanation for these findings is that LR after $\mathrm{BCT}$ is a marker for poor prognosis, as is the case with LR after mastectomy. ${ }^{12}$ At the time of our study, we did not encounter any mortality in patients with LR.

In our study, coincident LR and metastatic disease was noticed in one case. Local relapse as a predictor of distant metastasis was first described by Fisher et al.(1991). ${ }^{31}$ This was later confirmed by Whelan et al.(1994). ${ }^{30} \mathrm{~A}$ question regarding this correlation is if a local relapse after BCT is a causative of increased risk for distant metastasis and death? In other words: Do patients develop distant metastasis from their LR or is LR purely an indicator of aggressive disease? Unfortunately, this important question can not be answered from this or other studies. It may be that, after BCT, three situations can describe the relation of a LR and prognosis. In the first situation, a local relapse is only a LR and no distant metastasis occurs; these patients can be cured with salvage therapy. The second situation describes LR as part of a systemically metastasized disease. Therefore, LR can be seen as a marker of poor prognosis because LR, in this situation, is a precursor for distant metastasis. The third situation is feared most by clinicians, and describes local relapse as the cause of distant metastasis. This last situation is to be prevented at all costs. ${ }^{15}$ These effects were not detected in our study because of a small number of LR coexisting with distant metastasis (one case).

In conclusion, breast conservative therapy is an appropriate primary therapy for early breast cancer. We have shown LR rates 
comparable to those in other series. Radiation therapy was given adequately and did not influence the local relapse rate. The majority of recurrences detected were true or marginal recurrences, which arise mostly in the first 5 years after BCT. Follow-up of patients after BCT seems necessary certainly in these years. LR risk decreases gradually with increasing age, which makes it necessary to take in account the patient's age with the other known risk factors for LR, such as histopathological positive margins, to decide if a patient is suitable for breast-conserving therapy.

In the future, a detection of biological risk factors for types of LR would be useful to distinguish types of LR that are associated with distant metastasis and mortality. In that case, systemic therapy could be considered and, thus, improving a patient's distant metastasisfree survival and favorable LRs could be treated only locally.

\section{References:}

1- Veronesi U, Cascinelli N, Mariani L, et al: Twenty year follow up of a randomized study comparing breast conserving surgery with radical mastectomy for early breast cancer. N Engl J Med 2002; 347: 12271232.

2- Peters NH, Boel Rinkes LH, Zuithoff NP, et al: Meta-analysis of MR imaging in the diagnosis of breast lesions. Radiology 2007; 246 (1): 116-24.

3- Morakkabati N, Leutner CC, Schmiedel A: Breast MR imaging during or soon after radiation therapy. Radiology 2003; 229: 893-901.

4- Te Boekhorst DS, Peer NG, Van der Sluis RF et al: Periodic follow up after breast cancer and the effect on survival. Eur $J$ Surg 2001; 167: 490-496.

5- Recht AR, Connolly JL, Schnitt SJ, et al: The effect of young age on tumor recurrence in the treated breast after conservative surgery and radiotherapy. Int J Radiat Oncol Biol Phys 1988; 14: 3-10.

6- Gage I, Schnitt SJ, Nixon AJ, et al: Pathological margin involvement and the risk of recurrence in patients treated with breast-conserving therapy. Cancer 1996; 78: 1921-1928.
7- Leopold KA, Recht A, Schnitt SJ, et al: Results of conservative surgery and radiation therapy for multiple synchronous cancers of one breast. Int J Radiat Oncol Biol Phys 1989; 16: 11-16.

8- Voogd AC, Nielsen M, Peterse JL, et al: Differences in risk factors for local and distant recurrence after breast-conserving therapy or mastectomy for stage I and II breast cancer: pooled results of two large European randomized trials. J Clin Oncol 2001; 19: 1688-1697.

9 -Schnitt SJ, Abner A, Gelman R, et al: The relationship between microscopic margins of resection and the risk of local recurrence in patients with breast cancer treated with breast-conserving surgery and radiation therapy. Cancer 1994; 74: 1746-1751.

10-Preda L, Villa G, Rizzo S, et al: Magnetic resonance mammography in the evaluation of recurrence at the prior lumpectomy site after conservative surgery and radiotherapy. Breast Cancer Research 2006; 8: R53.

11-Fisher B, Anderson S, Redmond CK, et al: Reanalysis and results after 12 years of follow-up in a randomized clinical trial comparing total mastectomy with lumpectomy with or without irradiation in the treatment of breast cancer. $N$ Engl $J$ Med 1995; 333: 1456-1461.

12-McBain CA, Young EA, Swindell R, et al: Local recurrence of breast cancer following surgery and radiotherapy: Incidence and outcome. Clin Oncol R Coll Radioll 2003; 15(1): 25-31.

13-Toboul E, Buffat L, Belkacemi Y, et al: Local recurrences and distant metastases after breast conserving surgery and radiation therapy for early breast cancer. Int J Radiat Oncol Biol Phys 1999; 43: 25-38. 14-Fredriksson I, Liljegren G, Palm-Sjovall $\mathrm{M}$, et al: Risk factors for local recurrence after breast-conserving surgery. Br J Surg 2003; 90: 1093-1102.

15-Elkhuizen PH, van de Vijver MJ, Hermans $\mathrm{J}$, et al: Local recurrence after breastconserving therapy for invasive breast cancer: High incidence in young patients and association with poor survival. Int $J$ Radiat Oncol Biol Phys 1998; 40: 859-867. 
16-Gage I, Recht A, Gelman R, et al: Longterm outcome following breast-conserving surgery and radiation therapy. Int J Radiat Oncol Biol Phys 1995; 33: 245-251.

17-Schrading S, Kuhl K: Mammographic, US, and MR damaging phenotypes of familial breast cancer. Radiology 2008; 246(1): 5870.

18-Smith RA: The evolving role of MRI in the detection and evaluation of breast cancer. N Engl J Med 2007; 356 (13): 1367.

19-Kuhl CK, Schrading S, Leutner CC, et al: Mammography, breast ultrasound, and magnetic resonance imaging for surveillance of women at high familial risk for breast cancer. Journal of Clinical Oncology 2005; 33: 8469-8476.

20-Warner E, Plewes DB, Shumak RS: Comparison of breast magnetic resonance imaging, mammography and ultrasound for surveillance of women at high risk for hereditary breast cancer. J Clin Oncol 2001; 19: 3524-3531.

21-Osborne M, Borgen P, Wong G, et al: Salvage mastectomy for local and regional recurrence after breast-conserving operation and radiation therapy. Surg Gynecol Obstet 1992; 174: 189-194.

22-Osman MA, Halmy S, Al-Dory A, et al: Mammographic findings following breast conserving surgery and radiotherapy for carcinoma of female breast. Scientific journal of Al-Azhar Medical faculty 1994; 15(2): 497-506.

23-Borger J, Kemperman H, Hart A, et al: Risk factors in breast-conservation therapy. $J$ Clin Oncol 1994; 12: 653-660.

24-Harris JR, Connolly JL, Schnitt SS, et al: The use of pathological features in selecting the extent of surgical resection necessary for breast cancer patients treated by primary radiation therapy. Ann Surg 1985; 201: 164-169.
25-Mirza NQ, Vlastos G, Meric F, et al: Predictors of locoregional recurrence among patients with early stage breast cancer treated with breast conserving therapy. Ann Surg Oncol 2002; 9(3): 256265.

26-Broet P, de La Rochfordiere A, Scholl SM, et al: Analyzing prognostic factors in breast cancer using a multistate model. Breast Cancer Res Treat 1999; 54: 83-89.

27-Bartelink H, Horiot JC, Poortmans P, et al: European organization for research and treatment of cancer radiotherapy and breast cancer groups: Recurrence rates after treatment of breast cancer with standard radiotherapy with or without additional radiation. New Eng J Med 2001; 345: 13781386.

28-Freedman G, Fowble B, Hanlon A, et al: Patients with early stage invasive cancer with close or positive margins treated with conservative surgery and radiation have an increased risk of breast recurrence that is delayed by adjuvant systemic therapy. Int J Radiat Oncol Biol Phys 1999; 44: 10051015.

29-Chauvet B, Reynaud-Bougnoux A, Calais $\mathrm{G}$, et al: Prognostic significance of breast relapse after conservative treatment in nodenegative early breast cancer. Int J Radiat Oncol Biol Phys 1990; 19: 1125-1130.

30-Whelan T, Clark R, Roberts R, et al: Ipsilateral breast tumor recurrence postlumpectomy is predictive of subsequent mortality: Result from randomized trial. Int J Radiat Oncol Biol Phys 1994; 30: 1116.

31-Fisher B, Anderson S, Fisher ER, et al: Significance of ipsilateral breast tumor recurrence after lumpectomy. Lancet 1991; 338: 327- 331 . 